\section{The Moyal-Lie theory of phase space quantum mechanics}

To cite this article: T Hakioglu and Alex J Dragt 2001 J. Phys. A: Math. Gen. 346603

View the article online for updates and enhancements.

\section{Related content}

- The action-angle Wigner function

T Hakioglu and E Tepedelenlioglu

Quantum mechanics on the cylinder José A González, Mariano A del Olmo and Jaromir Tosiek

- Heisenberg evolution WKB and symplectic area phases

T A Osborn and M F Kondratieva

\section{Recent citations}

$\frac{\text { - QUANTUM CANONICAL }}{\text { TRANSFORMATIONS IN }}$
$\frac{\text { WEYL-WIGNER-GROENEWOLD-MOYA }}{\text { LFORMALISM }}$
$\frac{\text { TEKN DEREL et al }}{\text { - Weyl's symbols of Heisenberg operators of }}$
$\frac{\text { canonical coordinates and momenta as }}{\text { quantum characteristics }}$
M. I. Krivoruchenko and Amand Faessler
- STABILIZATION OF INTERNAL SPACE
$\frac{\text { IN NONCOMMUTATIVE }}{\text { MULTIDIMENSIONAL COSMOLOGY }}$
N. KHOSRAVI et al




\title{
The Moyal-Lie theory of phase space quantum mechanics
}

\author{
T Hakioğlu ${ }^{1}$ and Alex J Dragt ${ }^{2}$ \\ ${ }^{1}$ Physics Department, Bilkent University, 06533 Ankara, Turkey \\ ${ }^{2}$ Physics Department, University of Maryland, College Park, MD 20742-4111, USA
}

Received 9 March 2001

Published 17 August 2001

Online at stacks.iop.org/JPhysA/34/6603

\begin{abstract}
A Lie algebraic approach to the unitary transformations in Weyl quantization is discussed. This approach, being formally equivalent to the $\star$-quantization, is an extension of the classical Poisson-Lie theory and can be used as an efficient tool in the quantum phase space transformation theory.
\end{abstract}

PACS numbers: 02.20-a, 03.65-w, 45.20.Ji

The purpose of this paper is to show that the Weyl correspondence in the quantum phase space can be presented in a quantum Lie algebraic perspective, which in some sense derives analogies from the Lie algebraic approach to classical transformations and Hamiltonian vector fields. In the classical case transformations of functions $f(z)$ of the phase space $z=(p, q)$ are generated by the generating functions $\mathcal{G}_{\mu}(z)$. If the generating functions are elements of a set, then this set usually contains a closed subset which defines an algebra over the Poisson bracket (PB). To be more precise one talks about different representations of the generators and of the phase space algebras. The second well known representation is the so called adjoint representation of the Poisson algebra of the $\mathcal{G}_{\mu}$ in terms of the classical Lie generators $L_{\mathcal{G}_{\mu}}$ over the Lie algebra. These are the Hamiltonian vector fields with the Hamiltonian $\mathcal{G}_{\mu}$. By using the Liouville theorem, they characterize an incompressible and covariant phase space flow.

The quantum Lie approach can be based on a parallelism with the classical one above. In this case the unitary transformations are represented by a set of quantum generating functions $\mathcal{A}_{\mu}(z)$ which are the quantum partners of the $\mathcal{G}_{\mu}$. The non-commutativity is encoded in a new multiplication rule, i.e. $\star$-product. The quantum Lie generators $\hat{V}_{\mathcal{A}_{\mu}}$ over the Lie bracket (LB) are the adjoint representations of the generating functions $\mathcal{A}_{\mu}(z)$ over the Moyal bracket $(\mathrm{MB})$ and they correspond to quantum counterparts of the classical Hamiltonian vector fields $L_{\mathcal{G}_{\mu}}$. They are represented by an infinitely large number of phase space derivatives. The only exception is the generators of linear canonical transformations which define the $s p_{2}(\mathbb{R})$ sub algebra. In this case the generators are given by the classical Hamiltonian vector fields generated by quadratic Hamiltonians. 
The classical Lie approach is sometimes referred to as the Poisson-Lie theory (PLT). This terminology is quite appropriate for generalizations as the algebraic representations that are relevant are written by using the Poisson and Lie product rules. For a similar reason we suggest that the quantum approach be referred to as the Moyal-Lie theory (MLT).

In section 1 we start with a brief textbook discussion of the PLT. The Lie algebraic treatment of the quantum transformation theory starts in section 2 with a discussion of the Weyl correspondence (section 2.1) and the $\star$-product. The main results of this paper, the MLT and the $\star$-covariance, are discussed at length in section 2.2 . The associativity property is discussed comparatively with respect to the Poisson-Lie and Moyal-Lie algebras in section 3. In the light of covariance versus $\star$-covariance of the phase space trajectories under canonical transformations, the time evolution deserves specific attention. The time evolution in the MLT and its extended $\star$-covariance property is examined in section 4 . Finally, we discuss the equivalence of the MLT to the standard $\star$-quantization through the $\star$-exponentiation in section 5 .

\section{The Poisson-Lie theory and the classical phase space}

The classical Lie generators $L_{\mathcal{G}_{\mu}}$ are given by

$$
L_{\mathcal{G}_{\mu}}=\left(\partial_{z} \mathcal{G}_{\mu}\right)^{T} J \partial_{z}
$$

where the $\mathcal{G}_{\mu}=\mathcal{G}_{\mu}(z)$ are in the set of phase space functions (generating functions) where $\mu=1, \ldots, N$ describes the generator index, $N$ being the total number of generators, $z$ denotes the $2 n$-dimensional phase space row vector $z=\left(z_{1}, \ldots, z_{2 n}\right)=\left(q_{1}, \ldots, q_{n} ; p_{1}, \ldots, p_{n}\right)$, $\partial_{z}=\left(\partial_{z_{1}}, \ldots, \partial_{z_{2 n}}\right), T$ is the transpose of a row vector and $J$ is the $2 n \times 2 n$ symplectic matrix with elements $(J)_{i j}, i, j=(1, \ldots, 2 n)$

$$
(J)_{i j}= \begin{cases}1 & \text { if } j=i+n \\ -1 & \text { if } i=j+n \\ 0 & \text { all other cases. }\end{cases}
$$

The classical algebra of the phase space generating functions is defined over the PB

$$
\left\{\mathcal{G}_{\mu}, \mathcal{G}_{\nu}\right\}^{(P)}=\left(\partial_{z} \mathcal{G}_{\mu}\right)^{T} J\left(\partial_{z} \mathcal{G}_{\nu}\right)
$$

and that of the generators $L_{\mathcal{G}_{\mu}}$ over the LB

$$
\left[L_{\mathcal{G}_{\mu}}, L_{\mathcal{G}_{v}}\right] \equiv L_{\mathcal{G}_{\mu}} L_{\mathcal{G}_{v}}-L_{\mathcal{G}_{v}} L_{\mathcal{G}_{\mu}}=L_{\left\{\mathcal{G}_{\mu}, \mathcal{G}_{v}\right\}^{(P)}}
$$

where the LB is defined by the first equality.

A classical canonical transformation (CCT) is considered to be a symplectic phase space map $\mathcal{M}_{\vec{\epsilon}}: z \mapsto Z_{\vec{\epsilon}}(z)$ with $Z=\left(Q_{1}, \ldots, Q_{n} ; P_{1}, \ldots, P_{n}\right)$ describing the new set of canonical phase space pairs. The symplectic condition is the invariance of the PB given by

$$
\left\{z_{j}, z_{k}\right\}^{(P)}=\left\{Z_{j}, Z_{k}\right\}^{(P)}=J_{j k}
$$

which implies that $\mathcal{M}_{\vec{\epsilon}}$ is invertible. If the symplectic map is continuously connected to the identity within a domain $\vec{\epsilon}=\overrightarrow{0}$, i.e. $\mathcal{M}_{\vec{\epsilon}=\overrightarrow{0}}=\mathbb{I}: z \mapsto z$, Lie's first theorem [1] states that the first-order infinitesimal change in $z_{j}$ is

$$
\delta_{j}\left(z, \delta \epsilon^{(1)}\right)=\delta \epsilon_{\mu} L_{\mathcal{G}_{\mu}} z_{j}
$$

where a summation over the generating function index $\mu$ is assumed. Finite canonical transformations can be obtained by infinitely iterating equation (6) as

$$
Z_{j}=\exp \left\{\epsilon_{\mu} L_{\mathcal{G}_{\mu}}\right\} z_{j}
$$


and following $\left[L_{\mathcal{G}_{\mu_{k}}}, f\right]=\left\{\mathcal{G}_{\mu_{k}}, f\right\}^{(P)}$ and considering $f=z_{j}$,

$$
\begin{aligned}
Z_{j}=z_{j}+\epsilon_{\mu}\left\{\mathcal{G}_{\mu}, z_{j}\right\}^{(P)}+\frac{1}{2 !} \epsilon_{\mu_{1}} \epsilon_{\mu_{2}}\left\{\mathcal{G}_{\mu_{1}},\left\{\mathcal{G}_{\mu_{2}}, z_{j}\right\}^{(P)}\right\}^{(P)}+\cdots \\
+\frac{1}{k !} \epsilon_{\mu_{1}} \ldots \epsilon_{\mu_{k}}\left\{\mathcal{G}_{\mu_{1}},\left\{\ldots,\left\{\mathcal{G}_{\mu_{k}}, z_{j}\right\}^{(P)}, \ldots\right\}^{(P)}+\cdots\right.
\end{aligned}
$$

where summations over the repeated indices are assumed. Let us consider the case $N=1$. This allows us to simplify the notation and drop the index $\mu$. In fact, the generalization for finite $N$ is nontrivial because the generators $\mathcal{G}_{\mu}$ may be closed under a Poisson algebra. For what we present here such algebraic generalizations will not be needed. It can be shown that, if $f(z)$ has a convergent Taylor expansion in powers of $z$ in some domain $z \in \mathcal{D}$, the transformation of $f$ is

$$
f(Z)=\exp \left\{\epsilon L_{\mathcal{G}}\right\} f(z) \exp \left\{-\epsilon L_{\mathcal{G}}\right\}
$$

where it is to be noted that equation (9) is an operator relation, i.e. it can be multiplied on the left and/or right by any arbitrary function $g(z)$ without changing its validity. The transformed variable $Z$ and $f(Z)$ must also be well defined in the same domain $\mathcal{D}$. From equation (9) there follows the remarkable manifest covariance property [2,3]

$$
\left[\exp \left\{\epsilon L_{\mathcal{G}}\right\} f\right](z) \equiv f_{\epsilon}(z)=f\left(\left[\exp \left\{L_{\mathcal{G}}\right\} z\right]\right)=f(Z(z, \epsilon))
$$

and the generalized Leibniz rule

$$
\exp \left\{\epsilon L_{\mathcal{G}}\right\}[f(z) g(z)]=\left[\exp \left\{\epsilon L_{\mathcal{G}}\right\} f\right]\left[\exp \left\{\epsilon L_{\mathcal{G}}\right\} g\right]=f(Z) g(Z)
$$

which can be summarized in

$\exp \left\{\epsilon L_{\mathcal{G}}\right\}[f(z) \ldots]=\left[\exp \left\{\epsilon L_{\mathcal{G}}\right\} f(z)\right]\left[\exp \left\{\epsilon L_{\mathcal{G}}\right\} \ldots\right]=f(Z)\left[\exp \left\{\epsilon L_{\mathcal{G}}\right\} \ldots\right]$.

In equations (10) and (11) the square brackets indicate that the operator on the left acts on the object within the brackets only. In (12) the dots indicate the possibility of further functions to be acted upon. Although equations (10)-(12) are different ways of writing equation (9) we will keep them explicit for facilitating comparison with their quantum versions.

The MLT is a generalization of the PLT to the non-commutative phase space. It will be shown that the Moyal-Lie generators can be uniquely derived in the quantum case with similar covariance properties when the role of the ordinary functional product is taken by the $\star$-product. As it turns out, the manifest covariance property in equation (10) is modified because of the non-commutativity of the $\star$-product [4]. As opposed to the standard covariance in (10) under ordinary multiplication the new covariance rule is manifest under the $\star$-multiplication of the functions of the phase space. The classical covariance is then recovered in the $\hbar \rightarrow 0$ limit of the $\star$-covariance.

\section{Quantum Lie generators}

The quantum mechanical results presented here are corollaries of a recent work of one of the present authors [4] on the extended covariance in non-commutative phase space under canonical transformations ${ }^{2}$. Our formulation here is based on Weyl correspondence [5-7] as a consistent and analytic principle between the classical and the quantum phase spaces.

2 The breaking of the classical covariance is not realized in an arbitrary way (see section 2.2). Manifest invariance is realized when the ordinary product is replaced by the $\star$-product. Hence we consider this as an extension of the classical covariance. 


\subsection{The Weyl correspondence}

Let $\hat{z}=\left(\hat{z}_{1}, \ldots, \hat{z}_{2 n}\right)=\left(\hat{q}_{1}, \ldots, \hat{q}_{n} ; \hat{p}_{1}, \ldots, \hat{p}_{n}\right)$ describe the phase space operators satisfying, as usual, $\left[\hat{z}_{i}, \hat{z}_{j}\right]=i \hbar J_{i j}$ and zero for all other LBs (commutators). We adopt the same definitions for $z$ and $\partial_{z}$ as in the classical case. The Weyl map is a correspondence rule between the operators described by $\hat{\mathcal{F}}=\mathcal{F}(\hat{z})$ and the functions $f(z)$ denoted by $\hat{\mathcal{F}} \Leftrightarrow f$ as

$$
\hat{\mathcal{F}}=\int \mathrm{d} \mu(z) f(z) \hat{\Delta}(z) \quad f(z)=\operatorname{Tr}\{\hat{\mathcal{F}} \hat{\Delta}(z)\}
$$

where $\mathrm{d} \mu(z)=\prod_{k=1}^{n}\left[\mathrm{~d} q_{k} \mathrm{~d} p_{k} /(2 \pi \hbar)\right]$. Here $\hat{\Delta}(z)$ describes a mixed basis for operators and functions in the phase space given by

$$
\hat{\Delta}(z)=\int \mathrm{d} \mu(\omega) \mathrm{e}^{-\mathrm{i} \omega^{\mathrm{T}} J z / \hbar} \mathrm{e}^{\mathrm{i} \omega^{\mathrm{T}} J \hat{z} / \hbar}
$$

where $\omega=\left(\omega_{1}, \ldots, \omega_{2 n}\right)$ is in the same domain as $z$, i.e. $\mathbb{R}^{2 n}, \mathrm{~d} \mu(\omega)=$ $\prod_{k=1}^{n}\left[\mathrm{~d} \omega_{k} \mathrm{~d} \omega_{k+n} /(2 \pi \hbar)\right]$. For details on the properties of the continuous $\hat{\Delta}(z)$ basis we refer the reader to [4].

At a fundamental level the Weyl correspondence can be stated as

$$
\mathrm{e}^{\mathrm{i}(\omega J \hat{z}) / \hbar} \Leftrightarrow \mathrm{e}^{\mathrm{i}(\omega J z) / \hbar}
$$

for all real $\omega$. From equation (15) the association for the identity, i.e. $\mathbb{I} \leftrightarrow 1$, is unique for $\omega=0$. All other maps between the operators and the functions in the Hilbert space are derived from equation (15) by suitable differential operations. Equation (15) is an explicit rule which leaves the algebraic properties of operators in the phase space invariant. Let $\hat{\mathcal{F}} \leftrightarrow f$ and $\hat{\mathcal{G}} \leftrightarrow g$ be associated by the Weyl correspondence. Then

$$
\hat{\mathcal{F}} \hat{\mathcal{G}} \leftrightarrow f \star g
$$

where $\star$ is a non-commutative, i.e. $f \star g-g \star f \neq 0$, and associative, i.e. $f \star(g \star h)=(f \star g) \star h$, operation corresponding to the phase space image of the operator product. One way to define the $\star$-product is by its action explicitly on the functions of the phase space by

$$
f \star=f\left(z+\frac{\mathrm{i} \hbar}{2} J \overrightarrow{\partial_{z}}\right) \quad \star f=f\left(z-\frac{\mathrm{i} \hbar}{2} J \overleftrightarrow{\partial_{z}}\right)
$$

where the arrows indicate the action of the derivatives. We explicitly write the correspondence for the LB as

$$
[\hat{\mathcal{F}}, \hat{\mathcal{G}}] \Leftrightarrow f \star g-g \star f=\{f, g\}^{(M)}
$$

where the superscript $M$ denotes the MB defined by the well known expression

$$
\{f, g\}^{(M)}=\mathrm{i} \hbar\{f, g\}^{(P)}+\left.\mathcal{O}\left(\hbar^{k}, \partial_{z}^{(k)}\right)\right|_{3 \leqslant k} .
$$

The first term is i $\hbar$ times the $\mathrm{PB}$ as defined in equation (3). $\mathcal{O}\left(\hbar^{3}, \partial_{z}^{(3)}\right)$ represents higher-order terms which appear in infinitely odd powers of both $\hbar$ and $\partial_{z}$.

\subsection{The unitary transformations in phase space and quantum Lie generators}

All invertible transformations (including non-unitary ones) can be formalized in the quantum phase space [4]. In this paper we will only consider the unitary ones. Let $\hat{\mathcal{F}}$ be an operator and $\hat{U}_{\mathcal{A}}=\mathrm{e}^{\mathrm{i} \gamma \hat{\mathcal{A}}}$ a one-parameter unitary transformation with $\gamma \in \mathbb{R}$. In the following we prove that the unitary transformation of $\hat{\mathcal{F}}$ in the operator space has a unique representation in the phase space given by

$$
\hat{\mathcal{F}}^{\prime}=\hat{U}_{\mathcal{A}}^{\dagger} \hat{\mathcal{F}} \hat{U}_{\mathcal{A}} \Leftrightarrow f^{\prime}(z)=\mathrm{e}^{\mathrm{i} \gamma \hat{V}_{\mathcal{A}}} f(z)
$$


where $\hat{\mathcal{F}} \Leftrightarrow f, \hat{\mathcal{F}}^{\prime} \Leftrightarrow f^{\prime}$ as usual and $\hat{V}_{\mathcal{A}}$ is a nonlinear and Hermitian generator associated with a real phase space generating function $\mathcal{A}(z)$ by $\hat{V}_{\mathcal{A}}=\mathcal{A} \star-\star \mathcal{A}$.

Since $\hat{\mathcal{A}}$ is considered to be Hermitian, it can be represented in a symmetrically ordered series in $\hat{z}$ as

$$
\hat{\mathcal{A}}=\mathcal{A}(\hat{z})=\sum_{n, m, r} a_{n, m, r} \hat{p}^{n} \hat{q}^{m} \hat{p}^{r}
$$

where $a_{n, m, r}$ are some real coefficients and we indicated the $\hat{p}, \hat{q}$ dependence explicitly for clarity. In order to prove equation (20) we start with

$$
\hat{\mathcal{F}}^{\prime}=\hat{\mathcal{F}}-\mathrm{i} \gamma[\hat{\mathcal{A}}, \hat{\mathcal{F}}]+\cdots+\frac{(-\mathrm{i} \gamma)^{k}}{k !} \underbrace{[\hat{\mathcal{A}}, \ldots,[\hat{\mathcal{A}}, \hat{\mathcal{F}}], \ldots]}_{k}
$$

and calculate the commutator in the $\mathcal{O}(\gamma)$ term. We use a particular phase space representation of $\hat{z}$ known as the Bopp shifts and given by $[4,9,10]$

$$
\hat{z} \hat{\Delta}(z)=\underbrace{\left[z-\frac{\mathrm{i} \hbar}{2} J \vec{\partial}_{z}\right.}_{\hat{z}_{L}} \hat{\Delta}(z) \quad \hat{\Delta}(z) \hat{z}=\underbrace{\left[z+\frac{\mathrm{i} \hbar}{2} \overrightarrow{\partial_{z}}\right]}_{\hat{z}_{R}} \hat{\Delta}(z)
$$

where, explicitly

$$
\hat{z}_{L}=\left(\begin{array}{l}
\hat{p}_{L} \\
\hat{q}_{L}
\end{array}\right)=\left(\begin{array}{l}
p-\frac{\mathrm{i} \hbar}{2} \overrightarrow{\partial_{q}} \\
q+\frac{\mathrm{i} \hbar}{2} \overrightarrow{\partial_{p}}
\end{array}\right) \quad \hat{z}_{R}=\left(\begin{array}{l}
\hat{p}_{R} \\
\hat{q}_{R}
\end{array}\right)=\left(\begin{array}{c}
p+\frac{\mathrm{i} \hbar}{2} \overrightarrow{\partial_{q}} \\
q-\frac{\mathrm{i} \hbar}{2} \overrightarrow{\partial_{p}}
\end{array}\right) .
$$

It is readily verified that

$$
\left[\hat{z}_{j_{L}}, \hat{z}_{k_{R}}\right]=0 \quad\left[\hat{z}_{j_{L}}, \hat{z}_{k_{L}}\right]=\mathrm{i} \hbar J_{j k}=\left[\hat{z}_{k_{R}}, \hat{z}_{j_{R}}\right]
$$

By repeatedly applying equations (23) to the commutator of equation (21) with $\hat{\Delta}$ one explicitly obtains

$$
[\hat{\mathcal{A}}, \hat{\Delta}]=\hat{V}_{\mathcal{A}} \hat{\Delta} \quad \hat{V}_{\mathcal{A}}=\sum_{n, m, r} a_{n, m, r}\left\{\hat{p}_{L}^{r} \hat{q}_{L}^{m} \hat{p}_{L}^{n}-\hat{p}_{R}^{n} \hat{q}_{R}^{m} \hat{p}_{R}^{r}\right\} .
$$

Remembering that $\hat{\mathcal{A}}$ in equation (21) is symmetrically ordered, equation (26) implies that for $f(z)$ arbitrary

$$
\hat{V}_{\mathcal{A}} f=\left[\mathcal{A}\left(\hat{z}_{L}\right)-\mathcal{A}\left(\hat{z}_{R}\right)\right] f=\mathcal{A} \star f-f \star \mathcal{A}=\{\mathcal{A}, f\}^{(M)}
$$

where equation (17) is used in the last part. Note that $\hat{V}_{\mathcal{A}}$ is an operator, composed of powers of $z, \partial_{z}$ in the way that the Bopp operators $\hat{z}_{L, R}$ depend upon, reproducing the action of the commutator $[\hat{\mathcal{A}}, \hat{\Delta}]$ in the phase space. By recursive application of $(26)$ the $k$ th-order commutator in equation (22) is represented by

$$
\underbrace{[\hat{\mathcal{A}}, \ldots,[\hat{\mathcal{A}}, \hat{\Delta}], \ldots]}_{k}=\left(\hat{V}_{\mathcal{A}}\right)^{k} \hat{\Delta} \quad \text { for all } \quad 0 \leqslant k .
$$

Exponentiating equation (28)

$$
\hat{\mathcal{U}}_{\mathcal{A}}^{\dagger} \hat{\Delta} \hat{\mathcal{U}}_{\mathcal{A}}=\mathrm{e}^{-\mathrm{i} \gamma \hat{V}_{\mathcal{A}}} \hat{\Delta} \text {. }
$$

We now use this result in the second equation in (13) for $\hat{\mathcal{F}}^{\prime} \Leftrightarrow f^{\prime}$

$$
f^{\prime}(z)=\operatorname{Tr}\left\{\hat{\mathcal{F}}^{\prime} \hat{\Delta}\right\}=\operatorname{Tr}\left\{\hat{U}_{\mathcal{A}}^{\dagger} \hat{\mathcal{F}} \hat{U}_{\mathcal{A}} \hat{\Delta}\right\}=\operatorname{Tr}\left\{\hat{\mathcal{F}} \hat{U}_{\mathcal{A}} \hat{\Delta} \hat{U}_{\mathcal{A}}^{\dagger}\right\}=\mathrm{e}^{\mathrm{i} \gamma \hat{V}_{\mathcal{A}}} f(z)
$$

Note that the unitary transformation of $\hat{\mathcal{F}}$ involves left and right multiplications by the transformation operator $\hat{U}_{\mathcal{A}}$ and its conjugate. Because of the associativity these operations 
commute. The same is observed also with the exponential phase space operator in equation (30). Using equation (27) we write

$$
\mathrm{e}^{\mathrm{i} \gamma \hat{V}_{\mathcal{A}}}=\mathrm{e}^{\mathrm{i} \gamma\left[\mathcal{A}\left(\hat{z}_{L}\right)-\mathcal{A}\left(\hat{z}_{R}\right)\right]}=\mathrm{e}^{\mathrm{i} \gamma \mathcal{A}\left(\hat{z}_{L}\right)} \mathrm{e}^{-\mathrm{i} \gamma \mathcal{A}\left(\hat{z}_{R}\right)}=\mathrm{e}^{-\mathrm{i} \gamma \mathcal{A}\left(\hat{z}_{R}\right)} \mathrm{e}^{\mathrm{i} \gamma \mathcal{A}\left(\hat{z}_{L}\right)} .
$$

Equation (30) is the simplest form of a canonical transformation acting on the functions of the phase space. Using equations (17) and (27) it can be put in the form

$$
\begin{aligned}
& \mathrm{e}^{\mathrm{i} \gamma \hat{V}_{\mathcal{A}}} f=\mathrm{e}^{\mathrm{i} \gamma\left[\mathcal{A}\left(\hat{z}_{L}\right)-\mathcal{A}\left(\hat{z}_{R}\right)\right]} f \\
&= f+\mathrm{i} \gamma(\mathcal{A} \star f-f \star \mathcal{A})+\frac{(\mathrm{i} \gamma)^{2}}{2 !}(\mathcal{A} \star \mathcal{A} \star f+f \star \mathcal{A} \star \mathcal{A}-2 \mathcal{A} \star f \star \mathcal{A})+\cdots \\
&= f+\mathrm{i} \gamma\{\mathcal{A}, f\}^{(M)}+\frac{(\mathrm{i} \gamma)^{2}}{2 !}\left\{\mathcal{A},\{\mathcal{A}, f\}^{(M)}\right\}^{(M)} \\
&+\cdots+\frac{(\mathrm{i} \gamma)^{k}}{k !} \underbrace{\left\{\mathcal{A}, \ldots,\{\mathcal{A}, f\}^{(M)} \ldots\right\}^{(M)}+\cdots}_{k} \\
&= \mathrm{e}^{\mathrm{i} \gamma(\mathcal{A} \star)} f \mathrm{e}^{-\mathrm{i} \gamma(\star \mathcal{A})}
\end{aligned}
$$

which can also be deduced from (31) using (25).

There is a unique correspondence between $\hat{V}_{\mathcal{A}}$ and the unitary transformation $\hat{U}_{\mathcal{A}}$ that it represents as illustrated by

$$
\begin{aligned}
& \begin{array}{llll} 
& f(z) & \stackrel{\text { Weyl }}{\Longleftrightarrow} & \hat{\mathcal{F}} \\
\mathrm{e}^{\mathrm{i} \gamma \hat{V}_{\mathcal{A}}} & \stackrel{1}{\Longleftrightarrow} & \hat{U}_{\mathcal{A}} & \stackrel{\mathbb{1}}{ }
\end{array} \\
& f^{\prime}=\mathrm{e}^{\mathrm{i} \gamma \hat{V}_{\mathcal{A}}} f \stackrel{\text { Weyl }}{\Longleftrightarrow} \quad \hat{\mathcal{F}}^{\prime} .
\end{aligned}
$$

In particular, if we consider two such transformations acting on the phase space, denoting the correspondence by $\mathcal{A}_{1} \longleftrightarrow \hat{V}_{\mathcal{A}_{1}}$ and similarly for $\mathcal{A}_{2}$, we find

$$
\left[\hat{V}_{\mathcal{A}_{1}}, \hat{V}_{\mathcal{A}_{2}}\right]=\hat{V}_{\left\{\mathcal{A}_{1}, \mathcal{A}_{2}\right\}}{ }^{(M)} \text {. }
$$

Furthermore, if the operators $\hat{A}_{k}$ are the generators of an abstract Lie algebra the corresponding phase space operators $\hat{V}_{\mathcal{A}_{k}}$ generate the adjoint representation in the phase space of the same Lie algebra.

It is clear that the $\hat{V}_{\mathcal{A}_{k}}$ are quantum analogues of the classical Lie generators $L_{\mathcal{G}_{k}}$ of CCTs. To demonstrate this we examine the quantum analogue of the classical covariance property in equations (9) and (10). Since the analogue of $\mathcal{M}_{\epsilon}$ is the exponentiated generator in equation (30) we start with that equation. Using the correspondence in equation (16) we find

$$
f^{\prime} \star g^{\prime}=\operatorname{Tr}\left\{\hat{F}^{\prime} \hat{G}^{\prime} \hat{\Delta}\right\}=\operatorname{Tr}\left\{\hat{U}^{\dagger} \hat{F} \hat{G} \hat{U} \hat{\Delta}\right\}=(f \star g)^{\prime} .
$$

This equation is the quantum analogue of (11) written more explicitly as

$$
\left[\mathrm{e}^{\mathrm{i} \gamma \hat{V}_{\mathcal{A}}} f\right] \star\left[\mathrm{e}^{\mathrm{i} \gamma \hat{V}_{\mathcal{A}}} g\right]=\left[\mathrm{e}^{\mathrm{i} \gamma \hat{V}_{\mathcal{A}}} f \star g\right] .
$$

Considering (36) for arbitrary $g$ the analogue of equation (12) is found as

$$
\left[\mathrm{e}^{\mathrm{i} \gamma \hat{V}_{\mathcal{A}}} f\right] \star \mathrm{e}^{\mathrm{i} \gamma \hat{V}_{\mathcal{A}}}=\mathrm{e}^{\mathrm{i} \gamma \hat{V}_{\mathcal{A}}} f \star .
$$

Equation (37) is converted into

$$
f^{\prime} \star=\mathrm{e}^{\mathrm{i} \gamma \hat{V}_{\mathcal{A}}} f \star \mathrm{e}^{-\mathrm{i} \gamma \hat{V}_{\mathcal{A}}}
$$

which is the quantum analogue of equation (9). We now examine the covariance properties under the action of $\mathrm{e}^{-\mathrm{i} \gamma \hat{V}_{\mathcal{A}}}$ in analogy with equation (10). Suppose that $f(z)$ is expanded in terms of binomials $p^{m} q^{n}$ as

$$
f(z)=\sum_{0 \leqslant(m, n)} f_{m, n} p^{m} q^{n}
$$


We use in (39) the fact that $p^{n+m}=p^{n} p^{m}=\left[p^{n} \star p^{m}\right]$ for all $m, n$ and similarly for powers of $q$. Hence $f$ in equation (39) is equivalently expressed as

$$
f(p, q)=\sum_{0 \leqslant(m, n)} f_{m, n} \underbrace{[p \star \cdots \star p]}_{m} \underbrace{[q \star \cdots \star q]}_{n} .
$$

In (40) note that the ordinary product is neither commutative nor associative with the $\star$-product. It can now be checked by using equations (30) and (38) that classical covariance described by equation (10) is violated

$$
f^{\prime}=\left[\mathrm{e}^{\mathrm{i} \gamma \hat{V}_{\mathcal{A}}} f\right](z) \neq f(Z)
$$

where $Z=Z(z)$ are the new canonical coordinates given by

$$
Z=\left[{ }^{\mathrm{i} \gamma} \hat{V}_{\mathcal{A}} z\right]
$$

We now trace back to the origin of the violation of the classical covariance in equation (41). To illustrate the point we adopt two distinct and simple cases. As the first example consider $f=p q$. Our goal is to look for what causes the effect $f^{\prime} \neq P Q$. By using the properties of the $\star$-product we first write $f=p \star_{q, p} q+\mathrm{i} \hbar / 2$. We now apply the transformation in equation (30). By equations (35)-(38) we find $f^{\prime}=P \star_{q, p} Q+\mathrm{i} \hbar / 2$. The only way in which $p q \mapsto P Q$ is when $\star_{q, p}=\star_{Q, P}$. If this were true the covariance would have been manifest, since we would have had $P \star_{q, p} Q=P \star_{Q, P} Q=P Q-\mathrm{i} \hbar / 2$. Then inserting this in $f^{\prime}$, we would have found $f^{\prime}=P Q$. As the second example we consider $f(p, q)=p^{2}=[p \star p]$. Repeating the same calculation here we find that $f^{\prime}=P \star_{q, p} P \neq P^{2}$. The equality, which indicates covariance would have been obtained if and only if $\star_{q, p}=\star_{Q, P}$.

These two typical examples demonstrate that the very intrinsic property of the $\star$-operation, non-invariance under a general canonical transformation, i.e. $\star_{q, p} \neq \star_{Q, P}$, is responsible for the loss of classical covariance in the quantum case. One exceptional case, in which $\star_{q, p}=\star_{Q, P}$ can be identified, corresponds to the group of linear canonical transformations, i.e. $S p_{2}(\mathbb{R})$ as given by

$$
\left(\begin{array}{l}
P \\
Q
\end{array}\right)=g\left(\begin{array}{l}
p \\
q
\end{array}\right) \quad g=\left(\begin{array}{ll}
a & b \\
c & d
\end{array}\right) \in S L_{2}(\mathbb{R}) .
$$

By conjugation

$$
\left(\begin{array}{l}
\partial_{P} \\
\partial_{Q}
\end{array}\right)=\left(g^{T}\right)^{-1}\left(\begin{array}{l}
\partial_{p} \\
\partial_{q}
\end{array}\right) \quad\left(g^{T}\right)^{-1}=\left(\begin{array}{cc}
d & -b \\
-c & a
\end{array}\right) .
$$

The invariance of the $\star$-product, i.e. $\star_{q, p}=\star_{Q, P}$, is guaranteed by the constant coefficients. We now demonstrate, for the linear canonical transformations, the manifestation of the classical covariance in equation (10) and for a more general case $f(p, q)=p^{m} q^{n}$. By using the associativity property of $\star_{q, p}$ we note that $p^{m} q^{n}=\left[p_{\star}^{m}\right]\left[q_{\star}^{n}\right]$ where $\left[x_{\star}^{k}\right]=[\underbrace{x \star x \star \cdots \star x}_{k}]$ with $\star=\star_{q, p}$. Since mixtures of the $\star$ and the ordinary product are not associative, one must perform them in the order which is denoted by the square brackets above. Namely, we first perform the calculations within each square bracket and then multiply them by using the ordinary multiplication. Denoting a generator of the linear canonical transformation $\Omega$ by $\hat{V}_{\Omega}$, we find that $f^{\prime}=P_{\star}^{m} \star\left\{\mathrm{e}^{\mathrm{i} \gamma \hat{V}_{\Omega}}\right\} \star^{-1}\left\{\mathrm{e}^{-\mathrm{i} \gamma \hat{V}_{\Omega}}\right\} Q_{\star}^{n}$ where we have $\star=\star_{q, p}$. Since $\star_{q, p}=\star_{Q, P}$ we have $\star\left\{\mathrm{e}^{\mathrm{i} \gamma \hat{V}_{\Omega}}\right\} \star^{-1}\left\{\mathrm{e}^{-\mathrm{i} \gamma \hat{V}_{\Omega}}\right\}=1$. Here $\star^{-1}$ is defined as the inverse of the star product which is well defined and corresponds to the complex conjugate of $\star$. We therefore have $f^{\prime}=\left[P_{\star_{P, Q}}^{m}\right]\left[Q_{\star_{\star P, Q}}^{n}\right]=P^{m} Q^{n}$ for all non-negative $m, n$. Hence, under linear canonical transformations we have the desired covariance

$$
\left[\mathrm{e}^{\mathrm{i} \gamma \hat{V}_{\Omega}} f\right](z)=f(Z)
$$


and all other classical properties in equations (10) and (9) can be easily derived for the linear canonical transformations here.

Note that the three quantum generators of the linear canonical algebra $s p_{2}(\mathbb{R})$ are given by

$$
\begin{array}{ll}
\mathcal{A}_{1}=-\frac{1}{4}\left(\hat{p}^{2}-\hat{q}^{2}\right) & \mapsto \hat{V}_{\mathcal{A}_{1}}=\frac{\mathrm{i} \hbar}{2}\left(p \partial_{q}+q \partial_{p}\right) \\
\mathcal{A}_{2}=\frac{1}{4}\left(\hat{p}^{2}+\hat{q}^{2}\right) & \mapsto \hat{V}_{\mathcal{A}_{2}}=\frac{-\mathrm{i} \hbar}{2}\left(p \partial_{q}-q \partial_{p}\right) \\
\mathcal{A}_{3}=\frac{1}{4}(\hat{p} \hat{q}+\hat{q} \hat{p}) & \mapsto \hat{V}_{\mathcal{A}_{3}}=\frac{\mathrm{i} \hbar}{2}\left(p \partial_{p}-q \partial_{q}\right) .
\end{array}
$$

The corresponding classical generators $L_{\mathcal{G}_{k}}$ are generated by the same quadratic polynomials $\mathcal{A}_{k}$ in (46). It is observed that the quantum generators are identical to the classical generators (up to an $i \hbar$ factor), i.e. $\hat{V}_{\mathcal{A}_{k}}=\mathrm{i} \hbar L_{\mathcal{G}_{k}}$. In [4] three types of quantum phase space generator, i.e. the generators of $s p_{2}(\mathbb{R})$, the gauge and the contact transformations, have been studied. For all three cases exact solutions are possible and the quantum generating functions are identical to the classical ones. These three generators therefore generate the same canonical algebra and the group of transformations in the classical and quantum phase spaces. No other class of transformations has been found yet having this universal property between the classical and quantum cases. In the light of these possibilities, it was suggested that the canonical algebra is spanned solely by these three classes of generators [8] which is an important issue yet to be settled.

The time evolution as a canonical transformation can also be represented in terms of the MLT. As in the classical case, the Hamiltonian function is the generator of time translations in the phase space. This will be examined in section 4 . We now examine the question of associativity in the classical and the quantum phase space algebras.

\section{Associativity and classical phase space}

The PB as an algebraic extension of the abstract LB is not associative [1]. One way to understand this is to construct a Poisson product $\times$, which can be defined as [1]

$$
\times=\frac{1}{2} \stackrel{\leftarrow}{\partial_{z}} J \overrightarrow{\partial_{z}}
$$

so that the PB is represented as

$$
\{g, f\}^{(P)}=g \times f-f \times g .
$$

The $\times$-product satisfies the left and right distributive and the scalar laws. Hence it is a linear algebra. Nevertheless it is non-associative, i.e. $g \times(f \times h) \neq(g \times f) \times h$, which can be checked quite easily.

Why is associativity so important in classical phase space? In pre-quantum-mechanical times the answer to this question would be in order to understand the classical phase space as a group manifold on which a theory of Lie transformations can be established. A number of attempts in this direction have been made in the past but the most serious considerations start after the advent of quantum mechanics and were inspired by it $[1,11]$.

From the classical point of view, the quantum mechanical $\star$-product can be realized as an associative non-commutative and $\hbar$-parametrized deformation of the $\times$-product in equation (47). We may write the $\star$-product in terms of the Poisson product as

$$
\star=\exp \{\mathrm{i} \hbar \times\}
$$

The associativity of the $\star$-product is clear by its analytic correspondence with the LB. It can be illustrative to demonstrate how associativity is manifested in the phase space directly. 
Using equation (25) we note that the left and right multiplication in the $\star$-product defined by equation (17) commute as

$$
[f \star, \star g]=f\left(z_{R}\right) g\left(z_{L}\right)-g\left(z_{L}\right) f\left(z_{R}\right)=0
$$

for any two functions $f$ and $g$. Now consider three such functions $f, g, h$ as

$$
[f \star h] \star g=g\left(z_{L}\right) f\left(z_{R}\right) h=f\left(z_{R}\right) g\left(z_{L}\right) h
$$

where we used (50) in the last step in (51). The last equality implies that the differential operator $f\left(z_{R}\right)$ acts on all functions on its right

$$
f\left(z_{R}\right) g\left(z_{L}\right) h=f\left(z_{R}\right)\left[g\left(z_{L}\right) h\right]=f\left(z_{R}\right)[h \star g]=f \star[h \star g] .
$$

Associativity of the $\star$-product is then proved by a comparison of equations (51) and (52). However in the case of the Poisson product the classical version of su is given by $[f \times, \times g] \neq 0$ for arbitrary $f, g$, causing equations (51) and (52) to be invalid.

The associativity of the $\star$-product, combined with its manifest covariance under unitary transformations described by equation (35), demonstrates that the $\star$-product is the algebraic partner in the quantum phase space of the ordinary functional product in the classical phase space [7]. The coexistence of the $\star$ and ordinary products such as in (40) is very commonly encountered when the unitary transformation of a function $f(z)$ is to be calculated. It was proved in equations (45) that, with the exception of $s p_{2}(\mathbb{R})$, an arbitrary transformation does not preserve the classical covariance property. Here, we have an example of that from time evolution.

\section{4. $\star$-covariant time evolution in the phase space}

In the standard formulations of quantum mechanics the transformations can be represented by their actions on the states or on the operators or some consistent mixture of both. For the specific case of the time evolution these are known as the Schrödinger, Heisenberg and interaction pictures respectively. We argue in the following in favour of adding to this gallery the phase space picture. One distinction of the phase space with respect to the other pictures is that it is essentially an operator picture. In this operator picture the quantum states that the phase space operators act on are represented by the density operator. The second distinction is that every admissible phase space operator (see section 2.1) is uniquely mapped onto an admissible phase space function and the operator product is uniquely mapped onto the $\star$-product. In this scheme, the density operator is uniquely mapped to a Wigner function. In the positivist point of view, the second distinction greatly facilitates the use of the conceptual tools to see the quantum dynamics as a classical dynamics on a deformed phase space [4,7]. On the other hand, the opposite view is also possible. A negativist view can be based upon concentrating on the difficulties in the computations imported by the new rule of functional multiplication, i.e. the $\star$-product. As it turns out, in the case when a transitive action of a transformation is involved, the classical and quantum cases significantly differ. A typical example is the time evolution.

The time evolution in the phase space is a clear picture allowing a comparative analysis of the classical and quantum dynamics given by the same Hamiltonian $\mathcal{H}_{t}(p, q)$. In the quantum case we assume that $\mathcal{H}_{t}(p, q)$ can be mapped to a quantum mechanical Hamiltonian operator $\hat{\mathcal{H}}_{t}$ through the Weyl correspondence, i.e. $\mathcal{H}_{t}(p, q) \Leftrightarrow \hat{\mathcal{H}}_{t}$. An arbitrary function $f$ of the dynamical variables $z$ will be denoted by $f_{t}^{(c \ell)}(z)$ and $f_{t}^{(q)}(z)$ as the classical and quantum solutions corresponding to $f$. To facilitate the comparison further, we also assume that at a 
certain initial time $t_{0}=0, f_{0}^{(c \ell)}(z)=f_{0}^{(q)}(z)=f_{0}(z)$. The classical time evolution can be calculated as

$$
f_{t}^{(c \ell)}(z)=\mathcal{T} \mathrm{e}^{\epsilon \int_{0}^{t} \mathrm{~d} t^{\prime} L_{\mathcal{H}^{\prime}}} f_{0}^{(c \ell)}(z)
$$

where $\mathcal{T}$ is the time ordering operator defined in the standard way by

$$
\mathcal{T} \mathrm{e}^{\epsilon \int_{0}^{t} \mathrm{~d} t^{\prime} L_{\mathcal{H}_{t^{\prime}}}}=1+\epsilon \int_{0}^{t} \mathrm{~d} t^{\prime} L_{\mathcal{H}_{t^{\prime}}}+\frac{1}{2 !} \epsilon^{2} \int_{0}^{t} \mathrm{~d} t^{\prime} L_{\mathcal{H}_{t^{\prime}}} \int_{0}^{t^{\prime}} \mathrm{d} t^{\prime \prime} L_{\mathcal{H}_{t^{\prime \prime}}} \ldots
$$

If equation (20) is considered for the quantum time evolution generated by $\hat{U}_{\mathcal{H}_{t}}$, then by equations (26)-(29), the finite time evolution is represented in the phase space by

$$
f_{t}^{(q)}(z)=\mathcal{T} \mathrm{e}^{-\frac{\mathrm{i}}{\hbar} \int_{0}^{t} \mathrm{~d} t^{\prime} \hat{V}_{\mathcal{H}^{\prime}}} f_{0}(z) \quad \hat{V}_{\mathcal{H}_{t^{\prime}}}=\mathcal{H}_{t^{\prime}} \star-\star \mathcal{H}_{t^{\prime}} .
$$

For Hamiltonians quadratic in $z$ equations (53) and (55) yield identical results. For such quadratic Hamiltonians $L_{\mathcal{H}}=\mathrm{i} \hbar \hat{V}_{\mathcal{H}}$ and hence the time evolution is covariant, i.e. $f_{t}(z)=$ $f_{0}\left(\mathcal{T} \mathrm{e}^{-\frac{i}{\hbar} \int_{0}^{t} \mathrm{~d} t^{\prime} \hat{V}_{\mathcal{H}^{\prime}}} z\right)$. The difference arises when the third- and higher-order terms in $z$ are present in the Hamiltonian and/or in $f_{0}$. For example consider the typical case of the quartic oscillator $\mathcal{H}=\mathcal{H}_{0}+\frac{\lambda}{4} q^{4}$ where $H_{0}=\left(p^{2}+q^{2}\right) / 2$. Using (55) we have

$$
\hat{V}_{\mathcal{H}}=-\mathrm{i} \hbar\left(p \partial_{q}-q \partial_{p}\right)+\mathrm{i} \hbar \lambda\left(q^{3} \partial_{p}-\left(\frac{\hbar}{2}\right)^{2} q \partial_{p}\right) \text {. }
$$

There is no known method of calculating the exact analytic forms of the time dependence of the phase space trajectories generated by equation (56). The series expansion generates highly nonlinear polynomials in increasing powers of $z$ at each order. A finite series of arbitrarily high orders can, in principle, be performed numerically for the binomials $p^{m} q^{n}$. Exact results can be achieved for the type of Hamiltonian $\mathcal{H}=p^{2} / 2+V(q)$ if a periodic kick is introduced in the interaction $V(q)$. The model we consider is the periodically kicked Hamiltonian

$$
\mathcal{H}(p, q)=\frac{p^{2}}{2}+\lambda \mathcal{V}(q) \delta_{T}(t)
$$

where $\delta_{T}(t)$ is the periodic delta-kick function with the period $T$. The quantum case for (57) is exactly solvable for arbitrary potentials $V(q)$ and the solution is identical to the classical case. We start with the classical one. The transformation induced by equation (57) in one full step is given in terms of a time-ordered product of two unitary Lie generators as

$$
\begin{aligned}
f_{n+1}^{(-)}(z) & =\mathrm{e}^{T L_{p^{2} / 2} \mathrm{e}^{\lambda L_{\mathcal{V}}} f_{n}^{(-)}(z)} \\
& =f\left(\mathrm{e}^{T L_{p^{2} / 2}} \mathrm{e}^{\lambda L_{\mathcal{V}}} z\right)=f\left(z_{n+1}^{(-)}\right)
\end{aligned}
$$

where the classical covariance condition is explicitly stated in the second part of the equation. The minus sign in the superscript indicates that the value of $f_{n}$ is calculated infinitesimally before the $n$th kick. The time dependence for $z_{n}$ is given by the standard result

$$
\tilde{p}_{n+1}^{(-)}=\tilde{p}_{n}^{(-)}+\kappa \mathcal{V}^{\prime}\left(q_{n+1}\right) \quad q_{n+1}=q_{n}-\tilde{p}_{n}^{(-)}
$$

with $\tilde{p}_{n}=T p_{n}$ and $\kappa=T \lambda$.

The quantum analogue of equation (58) in the operator space is the time-ordered quantum evolution operator

$$
\hat{U}_{\mathcal{H}}=\mathrm{e}^{-\frac{i}{\hbar} \frac{\hat{p}^{2}}{2}} \mathrm{e}^{-\frac{i}{\hbar} \lambda \mathcal{V}(\hat{q})}
$$

where $\mathcal{V}(\hat{q})$ is the operator corresponding to the potential $\mathcal{V}(q)$. The unitary Lie generators are determined by equation (55). Time ordering brings complications and therefore we explicitly derive $\hat{V}_{\mathcal{H}}$ from equations (20) and (29). Consider equation (30) and $\mathcal{A}=\mathcal{H}$ therein, where $\mathcal{H}$ 
is given by (57). Note that, because of the unitarity of the time evolution and the cyclicity of the trace, the time ordering is effectively reversed for $\hat{\Delta}$ by one round of cyclic permutation of the operators in (30). After permuting the $\hat{U}_{\mathcal{H}}$ one round, we have

$$
\begin{aligned}
\hat{\mathcal{U}}_{\mathcal{H}}^{\dagger} \hat{\Delta} \hat{\mathcal{U}}_{\mathcal{H}} & =\mathrm{e}^{\frac{i}{\hbar} \lambda \mathcal{V}(\hat{q})} \mathrm{e}^{\frac{i}{\hbar} \frac{\hat{p}^{2}}{2}} \hat{\Delta} \mathrm{e}^{-\frac{i}{\hbar} \frac{\hat{p}^{2}}{2}} \mathrm{e}^{-\frac{i}{\hbar} \lambda \mathcal{V}(\hat{q})} \\
& =\mathrm{e}^{\frac{i}{\hbar} \hat{V}_{p^{2} / 2}} \mathrm{e}^{\frac{i}{\hbar} \lambda \hat{V}_{\mathcal{V}}} \hat{\Delta}
\end{aligned}
$$

yielding

$$
\begin{aligned}
& \mathrm{e}^{-\frac{\mathrm{i}}{\hbar} \hat{V}_{\mathcal{H}}}=\mathrm{e}^{-\frac{\mathrm{i} T}{\hbar}\left(\frac{p^{2}}{2} \star-\star \frac{p^{2}}{2}\right)} \mathrm{e}^{-\frac{\mathrm{i}}{\hbar} \lambda(\mathcal{V}(q) \star-\star \mathcal{V}(q))} \\
& f_{n+1}^{(q)}(z)=\mathrm{e}^{-T p \partial_{q}} \mathrm{e}^{-\frac{\mathrm{i} \lambda}{\hbar}\left[\mathcal{V}\left(q-\frac{\mathrm{i} \hbar}{2} \partial_{p}\right)-\mathcal{V}\left(q+\frac{\mathrm{i} \hbar}{2} \partial_{p}\right)\right]} f_{n}^{(q)}(z) .
\end{aligned}
$$

By using $f_{n}^{(q)}(z)=z$ the transformation for the phase space coordinates is found to be

$$
p_{n+1}=p_{n}+\lambda \mathcal{V}^{\prime}\left(q_{n+1}\right) \quad q_{n+1}=q_{n}-T p_{n}
$$

which is identical to the classical trajectory given by equation (59) after redefining $p_{n}$ via $\tilde{p}_{n}=T p_{n}$. However we will keep the $\lambda$ and $T$ dependences as they appear in equation (63) for reasons to be clarified later.

The analogy with the classical trajectories is limited to equations (59) and (63) or to those obtained from equations (59) and (63) by linear canonical transformations. It was discussed in section 2.2 that such transformations create an equivalence class maintaining the covariance under time evolution.

It is expected that the time evolution is non-covariant for a general function of phase space. By direct inspection it is observed that polynomials $P_{r}(z)$ of degree $r \leqslant 2$ evolve identically in the classical and quantum cases. As a specific example we calculate the one-time-step quantum evolution of $f=p^{3} q$ which is of order $r=4$ in $z$. We find $\mathcal{O}\left(\hbar^{2}\right)$ difference between the non-covariant full quantum solution and the covariant classical part indicated by $c \ell$. The difference is

$$
\left[p^{3} q\right]_{n+1}^{(q)}-\left[p^{3} q\right]_{n+1}^{(c \ell)}=\left[p^{3} q\right]_{n+1}^{(q)}-p_{n+1}^{3} q_{n+1}=-\left.\frac{3}{2} \lambda \hbar^{2} \partial_{q}^{3} V(q)\right|_{q=q_{n+1}}
$$

where use has been made of $\left[p^{3} q\right]_{n+1}^{(c \ell)}=\left(p_{n+1}\right)^{3} q_{n+1}$, where the latter are, i.e. $p_{n+1}, q_{n+1}$, the same for the classical and the quantum cases. It turns out that a generic difference between the $(q)$ and $(c \ell)$ transformations of any function is always $\mathcal{O}\left(\hbar^{2}\right)$ or higher. One immediate observation in equation (64) is that it is no longer possible, even after suitable normalizations, to express $\left[p^{3} q\right]_{n+1}^{(q)}$ by the parameter $\kappa$. The reason is the broken classical covariance under time evolution. The second observation in equation (64) is that depending on the shape of the potential $V(q)$ the long-time behaviour of the classical and quantum solutions can be very different. If the third derivative of the potential is oscillatory, as in the standard map, the difference between the long-time averages of the classical and quantum solutions in equation (64) vanishes. For polynomial potentials the right-hand side in equation (64) is unbounded. In this case, and for sufficiently large $n$, the classical and the quantum solutions can differ significantly.

Another feature of non-covariance concerns the time evolution of dynamical systems that are related to each other by nonlinear canonical transformations. Such transformations may help in understanding fundamentally different quantum behaviour of the classically equivalent systems. As a typical case under the evolution by the Hamiltonian in (57) consider the gauge transformation

$$
q \mapsto Q=q+a p^{3} \quad p \mapsto P=p
$$


where $a$ is a real parameter. Because of the nonlinear momentum dependence the quantum evolution of the new pair described by $P_{n}, Q_{n}$ differs from its classical partner. For (65) only the transformation of $Q$ is nontrivial. It can be calculated as

$$
\begin{aligned}
Q_{n+1}^{(q)}-Q_{n+1}^{(c \ell)} & =a\left(\left[p^{3}\right]_{n+1}^{(q)}-\left[p^{3}\right]_{n+1}^{(c \ell)}\right) \\
& =a\left(\left[p^{3}\right]_{n+1}^{(q)}-p_{n+1}^{3}\right)=-\left.\hbar^{2} \frac{a \lambda}{4} \partial_{q}^{3} V(q)\right|_{q=q_{n+1}} .
\end{aligned}
$$

What (66) says is that not only the classical and the quantum solutions differ, but also the transformed and untransformed quantum solutions under equation (65).

\section{Equivalence to the $\star$-exponential formalism}

The quantum Lie generator formalism as outlined in section 2.3 can become an indispensable tool in the quantum phase space. The equivalence of the Lie generators to the quantization by the $\star$-exponential can be easily demonstrated.

Consider the unitary transformation $\hat{U}_{\mathcal{A}}=\mathrm{e}^{\mathrm{i} \gamma \hat{V}_{\mathcal{A}}}$. Expanding $\hat{U}_{\mathcal{A}}$ in power series of $\hat{\mathcal{A}}$ and applying the Weyl map $\hat{\mathcal{A}}^{r} \Longleftrightarrow \underbrace{\mathcal{A} \star \ldots \star \mathcal{A}}_{r}$ we find that

$$
\hat{\mathcal{U}} \Longleftrightarrow u=\mathrm{e}_{\star}^{\mathrm{i} \gamma \mathcal{A}}=1+\mathrm{i} \gamma \mathcal{A}+\frac{(\mathrm{i} \gamma)^{2}}{2 !} \mathcal{A} \star \mathcal{A}+\frac{(\mathrm{i} \gamma)^{3}}{3 !} \mathcal{A} \star \mathcal{A} \star \mathcal{A}+\cdots
$$

Equation (67) is the well known $\star$-exponential [7]. The $\star$-exponential can be used in the transformation of $f$ as

$$
f^{\prime}(z)=u^{(-1)} \star f \star u=\mathrm{e}_{\star}^{-\mathrm{i} \gamma \mathcal{A}} \star f(z) \star \mathrm{e}_{\star}^{\mathrm{i} \gamma \mathcal{A}} \quad \star=\star_{z} .
$$

This equation involves the $\star$-product of the $\star$-exponential and it's direct calculation is notoriously difficult in yielding analytically closed forms. It is already difficult to calculate the $\star$-exponential and it is proven so at the most basic level, the harmonic oscillator [7]. Using the MLT approach the harmonic oscillator solution can be derived quite effortlessly. The calculation can be found in [4]. By using (67) and the results in section $3.2 \mathrm{e}_{\star}^{\mathrm{i} \gamma \mathcal{A}_{\star}}$ in (68) can be expressed as

$$
\begin{aligned}
& \mathrm{e}_{\star}^{-\mathrm{i} \gamma \mathcal{A}} \star \mathrm{e}^{-\mathrm{i} \gamma(\mathcal{A} \star)}=\mathrm{e}^{-\mathrm{i} \gamma \mathcal{A}\left(\hat{z}_{R}\right)} \\
& \star \mathrm{e}_{\star}^{\mathrm{i} \gamma \mathcal{A}}=\mathrm{e}^{\mathrm{i} \gamma(\star \mathcal{A})}=\mathrm{e}^{\mathrm{i} \gamma \mathcal{A}\left(\hat{z}_{L}\right)} .
\end{aligned}
$$

If we further use equation (25) we observe that equation (69) is equivalent to $f^{\prime}=\mathrm{e}^{\mathrm{i} \gamma} \hat{V}_{\mathcal{A}} f$ as given by (30).

\section{Conclusion}

In this paper, we have introduced a new phase space approach to Weyl quantization referred to as the Moyal-Lie theory. MLT is basically a corrected PLT by the manifestation of associativity. The new approach is equipped with all other features of classical Hamiltonian vector fields. An exceptional case is the need for a new concept of covariance. It is shown here that the covariance is equipped in the non-commutative phase space with a star product. This suggest that one may still be able to define trajectories in the non-commutative sense.

The new theory is formally equivalent to the $\star$-quantization of Flato et al and Bayen et al [7] and it may be physically more appealing. This is partially due to its aspect which has to do with the algebraic similarities, in the classical Hamiltonian vector fields and the phase space Lie transformations. The second aspect is the connection with the theory of quantum canonical transformations in the phase space which is reported elsewhere [4]. 


\section{Acknowledgments}

TH is grateful to C Zachos (High Energy Physics Division, Argonne National Laboratory) for discussions. The support and the hospitality of University of Maryland is acknowledged.

\section{References}

[1] Santilli R M 1983 Foundations of Theoretical Mechanics II: Birkhoffian Generalizations of Hamiltonian Mechanics (Berlin: Springer)

[2] Deprit A 1969 Celest. Mech. 112

[3] Dragt A J and Finn J M 1976 J. Math. Phys. 172215

[4] Hakioğlu T 2000 Extended covariance under nonlinear canonical transformations in Weyl quantization Preprint ANL-HEP-PR-00-119

Hakioğlu T 2000 Preprint quant-ph/0011076

[5] Weyl H 1927 Z. Phys. 461

[6] von Neumann J 1931 Math. Ann. 104570

Wigner E P 1932 Phys. Rev. 40749

Groenewold H J 1946 Physica 12405

Moyal J E 1949 Proc. Camb. Phil. Soc. 4599

[7] Flato M, Lichnerowicz A and Sternheimer D 1975 J. Math. Phys. 171754

Bayen F, Flato M, Fronsdal C, Lichnerowicz A and Sternheimer D 1978 Ann. Phys., NY 110111

Bayen F, Flato M, Fronsdal C, Lichnerowicz A and Sternheimer D 1978 Ann. Phys., NY 11161

[8] Leyvraz F and Seligman T 1989 J. Math. Phys. 302512

[9] Bopp F 1956 Ann. Inst. H. Poincaré 1581

[10] Verçin A 1998 Ann. Phys., NY 266503

Dereli T and Verçin A 1997 J. Math. Phys. 385515

[11] Birkhoff G D 1927 Dynamical Systems (Providence, RI: American Mathematical Society)

[12] Casati G, Chirikov B V, Guarneri I and Shepelyansky D L 1979 Phys. Rep. 15477 